\title{
Photonic Crystal and its Application as a Biosensor for the Early Detection of Cancerous Cells
}

\author{
https://doi.org/10.3991/ijoe.v16i03.12523 \\ Anthony U. Adoghe, Etinosa Noma-Osaghae ( $\bowtie)$, \\ Yabkwa Rimamchika Israel \\ Covenant University, Ota, Nigeria \\ etinosa.noma-osaghaedcovenantuniversity.edu.ng
}

\begin{abstract}
The motivation for this paper is the strikingly sad statistics, obtained from various research bodies globally, regarding the effect of cancers on the global populace and the impact of current methods put in place for the early diagnosis of cancer. This paper is novel for many reasons. Primarily, it presents the use of an optical biosensor based on photonic crystal for cancer cell detection. This biosensor was recently developed as an ultra-compact biochemical sensor based on a $2 \mathrm{D}$ photonic crystal cavity known for altering its spectrum in proportion to minute changes in refractive index. Secondarily, The Finite Difference Time Domain (FDTD) and Plane Wave Expansion (PWE) techniques were applied to analyze the possibility of using photonic crystals as biosensors for the detection of cancer cells. The obtained resonant wavelength from the analysis of simulated results was $1.54964 \mu \mathrm{m}$ and transmitted power obtained from the analysis was $51.9 \%$. For the cancerous cell sample, The PC 12 Cell, the obtained resonant wavelength from analysis of the simulated results was $1.54964 \mu \mathrm{m}$ and the transmitted power obtained from the analysis was 55.6\%.
\end{abstract}

Keywords-Biosensors; Cancer; Finite Difference Time Domain; Plane Wave Expansion; Photonic crystal; refractive index.

\section{Introduction}

A biosensor is a biological analytic device that coverts a biological response into a measurable signal. Photonics is an improvement or advancement in the branch of optical technology concerned with the properties and transmission of photons or light. To apply photo crystals as sensors, certain characteristics of light, such as refractive index is observed as it changes in different environments.

The analysis of the blood assumes a vital place for the detection and prevention of hematological disorders. Blood disorders cause numerous Non-Communicable Diseases (NCDs), among which are, cardio-vascular sicknesses, malignancies, diabetes and respiratory infections [1] [3]. NCDs are responsible for 36 million deaths in the world every year, as revealed by the World Economic Forum and the Harvard School [2] [4]. The dielectric parameters of blood are of incredible significance for different restorative applications. For example, malignancy cell recognition, di-electric coag- 
ulometry and understanding physical, organic and compound properties of various blood constituents [1] [5]. The world health Organization WHO has acclaimed cancer to be the second major antecedent of death worldwide, accounting for nearly 1 in every 6 deaths [2] [6]. This very threatening disease is usually caused by metastasis, which refers to the rapid, abnormal and uncontrollable growth and reproduction of body cells. Cancers are usually difficult to detect because symptoms begin to show only at the final stage of maturity. Notwithstanding, mortality rate can be reduced if cancerous cells are detected early enough.

In this paper, focus is placed on the use of photonic crystals to detect cancer cells. An example of a very common type of cancer is the cervical cancer. Its symptoms include bleeding in between periods and after sexual intercourse. Foul smelling white discharge and low back pain or lower abdominal pain may also occur. Some devices have been developed to enable medical practitioners detect cancer cells in the body of patients. One of these devices are biosensors. Figure 1 shows the difference between cancerous and normal cells.

The problem this study wants to solve are the current limitations in conventional methods for early cancer detection. A number of factors that increase the risk of error in the early detection of cancer cells in patients using conventional techniques have been identified. Some of these limitations are, the threat posed by the use of $\mathrm{x}$-rays for imaging in which if done often and at high frequency can cause cancer in patients. Sometimes, the results obtained from microscopic analysis may vary from one oncologist or technician to another. Depending on pathologist or operator. This raises doubts in the confidence of patients in the expertise of oncologist and medical experts.

Colposcopy impressions and human papillomavirus genotyping has pre-cancer sensing accuracy within 2-82\% across 24 strata [7]. Also, most of the conventional methods of cancer cell detection take hours to days before test results become available. This is a serious limitation because as earlier stated, cancer is treatable if it is detected early.

Another problem is the size of the currently available equipment or devices used for cancerous or pre-cancerous cell detection [8]. Many of the existing equipment require very large facilities and laboratories for detection or diagnostics. This means that it will be difficult for patients to readily do a self-test when they feel some discomfort.

Intrusion of privacy is an issue faced by conventional methods of cancer cell detection. Cervical cancer detection requires such clinical processes like colposcopy to confirm its presence [9]. In a study by [9], only $60.8 \%$ of the women were comfortable with invasive medical processes needed to detect the presence of cancerous cells.

The remaining part of the paper is organized this way. Section two gives an overview of the existing literature on the use of photonic crystals as biosensors. Section three elucidates the methodology used to achieve the objective of adapting the photonic sensor based biosensor for early cancer cell detection. Section four provides a discussion of the results obtained from the study. A conclusion is made along with some acknowledgements and recommendations for future work. 


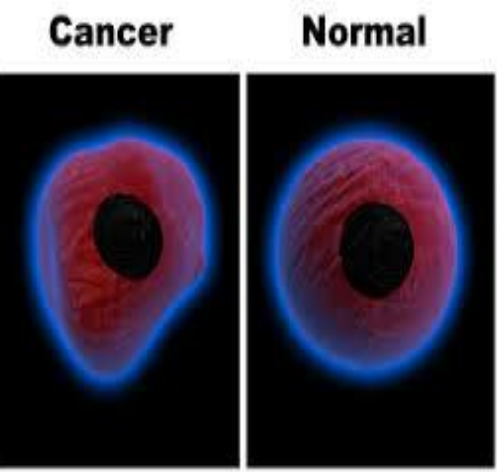

Fig. 1. Normal and Cancerous Cell

\section{Review of Related Works}

Wentzensen et al [7], evaluated if multi- aimed cervical biopsies on the basis of screening cytology, human papillomavirus testing, and colposcopy impression has the ability to make screening results better. It was however discovered that colposcopy impression enables better recognition of precancerous cervical cells. Kowalik et al [10], provided an overall review of CTC phenotypes. The authors reviewed existing literature on detecting CTC cervical precancer. Cervical cytology, human papillomavirus genotyping, and colposcopy impression were used to establish up to 24 different risk strata. Results show that accuracy of detecting precancer cells ranged from $2-82 \%$ across 24 strata based on colposcopy detection methodology.

Chigbu and Aniebue [9] evaluated the overall experience and recommendations of women who took colposcopy in south eastern Nigeria by interviewing women using structured interviewer-administered questionnaires. The most dissatisfying aspect of colposcopy reported was privacy intrusion. Conversely, when clinic personnel reassure patients, satisfaction soars. Higher proportion were more comfortable with female colposcopist according to studies.

Photonic crystal based optical sensors work based on the resonance wavelength principle [11]. Based on this sensing principle, any mechanical deformation caused from within or without the sensor, the resonance wavelength or output intensity of the sensor will vary, thereby showing sensitivity towards effects triggered internally or externally.

The wavelength at which the sensor operates, where the highest point occurs in the spectrum's normalized output is what is referred to as resonant wavelength [12]. The symbol $\lambda_{0}$ represents it. Bandgap of photonic optical sensor depends on the three main designing parameters named as lattice constant of crystal structure, refractive index of sensor material, radius to lattice constant ratio (r/a) of unit cell. At any time when there is an external effect on the sensor structure, there is a corresponding variation in the sensor parameters [13] [14] which causes an alteration in the band gap of the sensor and as such shifts the resonant wavelength of the biosensor. The analysis of 
the performance parameter [15] is done with the Quality Factor [16]. The Quality Factor is the ratio of resonance frequency to the full width at half maxima (FWHM) of the resonator's Lorentzian response represented as $\omega 0 / \Delta \omega$. The quality factor is represented as ' $Q$ '.

Table 1. Parameter Specifications of the Proposed Photonic Crystal Based Biosensor.

\begin{tabular}{|l|c|}
\hline \multicolumn{1}{|c|}{ Name of Parameter } & Values \\
\hline Radius of the hole (r) & $0.1385 \mu \mathrm{m}$ or 138nm \\
\hline Lattice Constant (a) & $0.400 \mu \mathrm{m}$ or 40nm \\
\hline Background Index & Silicon $(\mathrm{n}=3.50)$ \\
\hline Refractive Index (holes) & Air $(\mathrm{n}=1)$ \\
\hline Refractive index of normal cell & 1.350 \\
\hline Refractive index of Jurket cell & 1.390 \\
\hline Refractive index of PC 12 cell & 1.395 \\
\hline Refractive index of MDA MB231 cell & 1.399 \\
\hline Refractive index MCF 7 cell & 1.401 \\
\hline PBG range & $1203 \mathrm{~nm}-61812 \mathrm{~nm}$ \\
\hline Polarization & TM \\
\hline Size & $8 \mu \mathrm{m} \times 6.65 \mu \mathrm{m}$ \\
\hline
\end{tabular}

\section{Methods}

In this study, a photonic crystal-based biosensor for the detection of cancer cells was designed. The sensor design includes the linear waveguide with the Nano-cavity. The Biosensor was designed using hexagonal structure with the lattice constants; $\mathrm{a}=$ $0.40 \mu \mathrm{m}$, the radius of air holes' $\mathrm{r}=0.138 \mu \mathrm{m}$, and the refractive index of silicon slab was 3.50. Observations showed that different cells have different refractive index and these difference in refractive index, shifts the intensity of the resonant wavelength of the sensor. In this sensor when there are no sample at the suspected region, the resonance wavelength was $1.550 \mu \mathrm{m}$. The designed sensor mainly works on the short band.

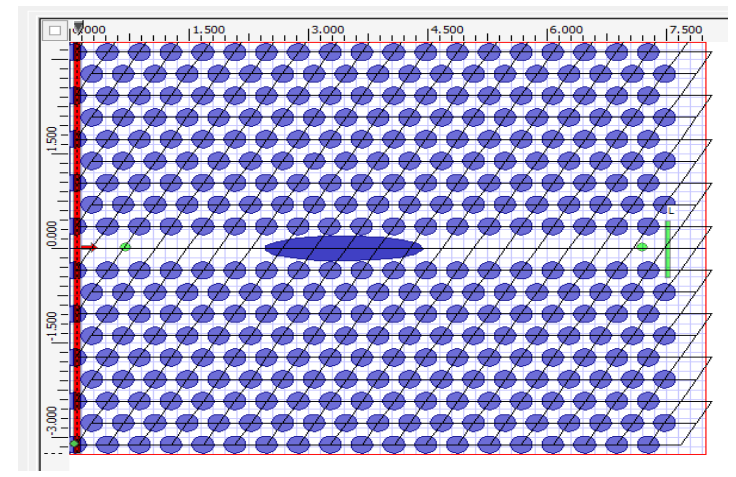

Fig. 2. 2D photonic crystal layout 
From Figure 2, it is clearly seen that there are two holes in the middle of the proposed structure. The cells are placed into the hole for sensing [1]. The outputs from the simulations were compared and to detect which cell was cancerous. The cancerous or malignant cells as they are also called have much higher refractive indices as compared to normal cells [17]. This certainly affects the simulation outputs and further calculations.

\section{Results}

The interpretation of the results of the simulation were got via observation. There were two observation points for the simulation; observation (s-band) and conventional band (c-band). It is known that cancer cells have higher levels of acidity than normal cells, hence different refractive index [1]. The sensor design consists of a 2dimensional hexagonal array of dielectric air in silicon wafer with two-point defects. The two air holes in the silicon wafer along the line of defect contain the cells to be tested. The light passed through the photonic crystal from one end will interact with the cell in the holes and compare their outputs, depending on the refractive index of the cells which have different levels of acidity [8]. Point 1 was for the input electromagnetic waves. Observation point 2 was for the output. The results were derived using the Fast Fourier Transform (FFT) of the second point of observation. This was between the wavelengths of $1.4 \mathrm{um}$ to $1.6 \mathrm{um}$. The Discrete Fourier Transform (DFT) could also be obtained. Table 1 shows the various values that can be obtained for different measurement parameters and for different cell type.

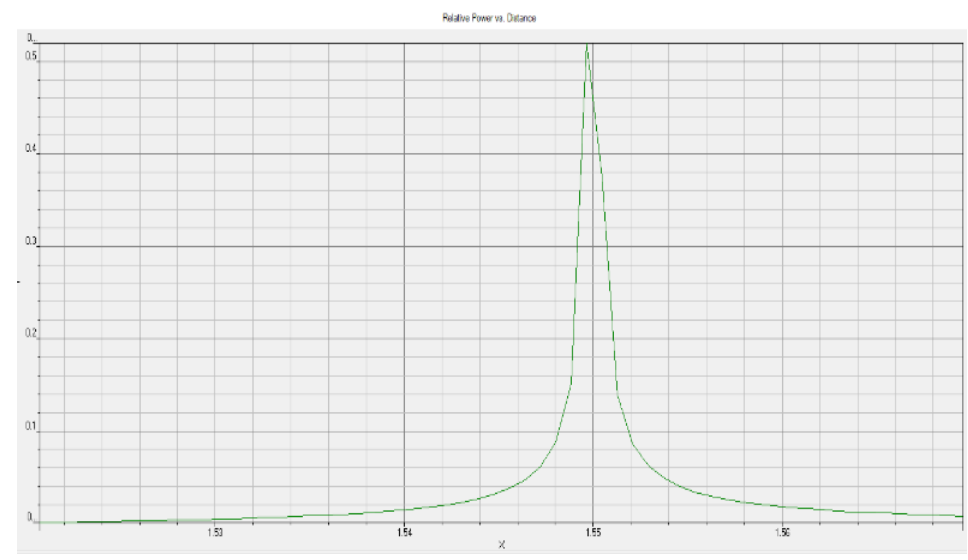

Fig. 3. Normalized transmission spectrum of Normal Cell

From our Table 2 it can be seen that as the refractive index of material or the blood sample increases there is a corresponding increase in the transmission of light through the sensor. So, the sensor senses the changes in the molecules by their varying refractive index. 
The simulation ran for 100000-time steps at InputPlane1 and a wavelength of 1.550 um. The analysis of the quality factor was obtained from the ratio of the resonant frequency and the full width at half maxima. The quality factor gave an indication of the performance of the system. The table below shows the quality factor as calculated along with other parameters as refractive index and the transmission according to cell type.

Figure 3 displays the normalized transmission spectrum of the sensor for Normal Cell. This normal cell refers to the normal blood sample without any metastasis. The obtained resonant wavelength from the analysis of simulated results was $1.54964 \mu \mathrm{m}$ and transmitted power obtained from the analysis was $51.9 \%$. Table 2 represents.

Figure 4 displays the transmission spectrum of sensor for cancerous Cell [pc12]. This cell refers to the cancerous blood sample having metastasis. In this case, the obtained resonant wavelength from analysis of the simulated results was $1.54964 \mu \mathrm{m}$ and the transmitted power obtained from the analysis was $55.6 \%$.

\section{Discussion}

Figure 5 to Figure 6 shows the propagation of light wave with its different colors through the photonic crystal and how it resonated with the cavity in the 2-dimensional magnetic field distribution. It can be clearly seen that the initial intensity of light entering the sensor is not the same as that at the output observation point. There is a depletion or reduction of intensity caused by the operation taking place in the cavity of the sensor. The light propagated resonates with the blood samples and gives an output based on the different refractive index each blood sample possesses.

This form of diagnostic bio-photonics can be used to detect diseases in their initial stages before actual medical symptoms occur in patients. By using optics, diagnostic bio-photonics provides several advantages of sensing and imaging at the molecular level and also collects multidimensional data for evaluation. Technologies based on light are generally contact-free with less effect on the integrity of living subjects and consequently, can easily be applied to the visualization of complex structures, cellular level diagnosis and lots more.

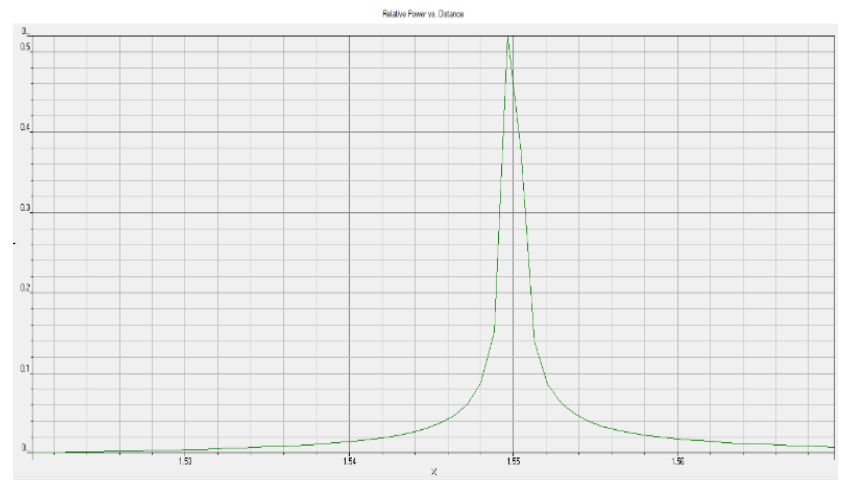

Fig. 4. Transmission spectrum of one of the cancerous Cell [pc12]. 


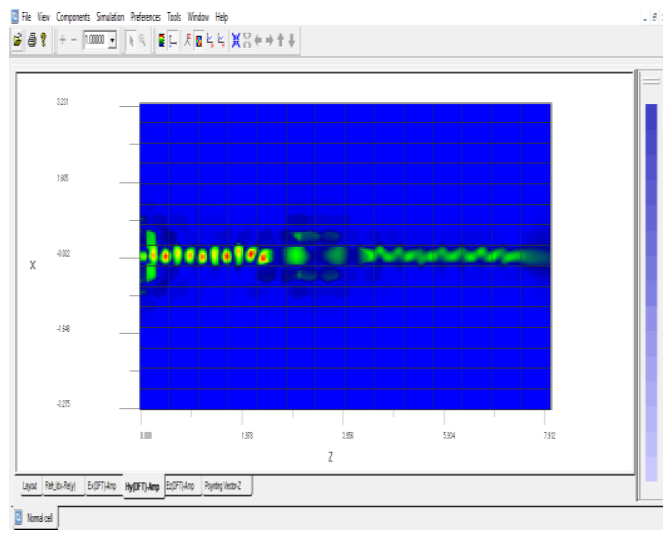

Fig. 5. Normal blood cell magnetic field distribution in 2D.

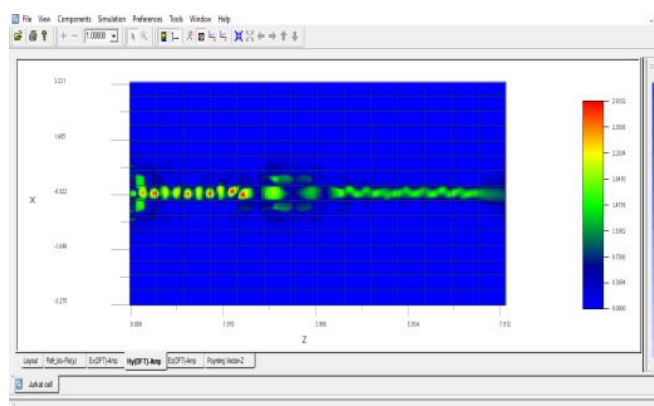

Fig. 6. MC7 cell magnetic field distribution in 2D.

Table 2. Measured Parameters of Different Cells using the Photonic Crystal Based Biosensor.

\begin{tabular}{|l|c|c|c|}
\hline \multicolumn{1}{|c|}{ Name of Cell } & Effective Refractive Index & Resonant Wavelength & Transmission power (\%) \\
\hline Normal Cell & 1.350 & 1.54966 & 51.9 \\
\hline Jurket Cell & 1.390 & 1.54966 & 55.1 \\
\hline PC-12Cell & 1.395 & 1.54966 & 55.6 \\
\hline MDA MB 232 Cell & 1.399 & 1.54966 & 56.0 \\
\hline MCF 7 Cell & 1.350 & 1.54966 & 51.9 \\
\hline
\end{tabular}

\section{Conclusion}

The aim of the study, which was to design a 2-dimensional photonic crystal-based biosensor for the early detection of cancer cells was achieved. The proposed design was efficient because of its smaller size, lower cost, less losses and compact nature. The limitation of the proposed design was its inability to test more than cell at a time. The other cell being a sample of the normal one. The Plane wave expansion was employed for simulation in the Finite Difference Time Domain (FDTD) tool. An improvement should be made in the sensing technique. This will enable detection of 
different samples at one time. The sensor should be modified to have a better qualityfactor. This encourage its fabrication and commercialization.

\section{$7 \quad$ Acknowledgement}

This paper is sponsored by Covenant University, Ota, Ogun State, Nigeria.

\section{$8 \quad$ References}

[1] P. Sharma, P. Sharan, and P. Deshmukh, "A photonic crystal sensor for analysis and detection of cancer cells," in 2015, International Conference on Pervasive Computing (ICPC), 2015, pp. 1-5. https://doi.org/10.1109/pervasive.2015.7087208

[2] "Cancer Fact Sheet", 2018

[3] E. M. Dewi, E. Purwanti, and R. Apsari, "Cervical Cell Classification using Learning Vector Quantization (LVQ) Based on Shape and Statistical Features," International Journal of Online Engineering, vol. 15, no. 2, 2019. https://doi.org/10.3991/ijoe.v15i02.9796

[4] E. Purwanti and I. S. Preswari, "Early Risk Detection of Pre-eclampsia for Pregnant women using Artificial Neural Network," International Journal of Online Engineering, vol. 15, no. 2, 2019. https://doi.org/10.3991/ijoe.v15i02.9680

[5] O. Thinnukool, P. Khuwuthyakorn, P. Wientong, B. Suksati, and N. Waisayanand, "Type 2 Diabetes Mobile Application for Supporting for Clinical Treatment: Case Development Report," International Journal of Online Engineering, vol. 15, no. 2, 2019. https://doi.org/ 10.3991/ijoe.v15i02.9769

[6] H. H. Muljo, A. S. Perbangsa, and B. P. Yulius, "Improving Early Cancer Detection Knowledge through Mobile Learning Application," iJOE, vol. 15, no. 2, p. 61, 2019. https ://doi.org/10.3991/ijoe.v15i02.9678

[7] N. Wentzensen, J. Walker, K. Smith, M. A. Gold, R. Zuna, L. S. Massad, et al., "A prospective study of risk-based colposcopy demonstrates improved detection of cervical precancers," American Journal of Obstetrics and Gynecology, vol. 218, pp. 604.e1-604.e8, 2018/06/01/ 2018. https://doi.org/10.1016/j.ajog.2018.02.009

[8] V. Sharma, V. L. Kalyani, and S. Upadhyay, "Photonic crystal based bio-sensor detection in cancer cell using FDTD method," in 2017 8th International Conference on Computing, Communication and Networking Technologies (ICCCNT), 2017, pp. 1-5. https://doi.org/ $\underline{10.1109 / \text { iccent.2017.8204043 }}$

[9] C. O. Chigbu and U. U. Aniebue, "Experiences of women undergoing colposcopy in southeastern Nigeria," International Journal of Gynecology \& Obstetrics, vol. 119, pp. 182-184, 2012/11/01/ 2012. https://doi.org/10.1016/j.ijgo.2012.06.005

[10] A. Kowalik, M. Kowalewska, and S. Góźdź, "Current approaches for avoiding the limitations of circulating tumor cells detection methods-implications for diagnosis and treatment of patients with solid tumors," Translational Research, vol. 185, pp. 58-84. e15, 2017/07/01/ 2017. https://doi.org/10.1016/j.trsl.2017.04.002

[11] T. Baba, N. Fukaya, and J. Yonekura, "Observation of light propagation in photonic crystal optical waveguides with bends," Electronics Letters, vol. 35, pp. 654-655, 1999. https:// doi.org/10.1049/el:19990438

[12] S. Jindal, S. Sobti, M. Kumar, S. Sharma, and M. K. Pal, "Nanocavity-Coupled Photonic Crystal Waveguide as Highly Sensitive Platform for Cancer Detection," IEEE Sensors Journal, vol. 16, pp. 3705-3710, 2016. https://doi.org/10.1109/jsen.2016.2536105 
[13] M. P. Ajayi, E. O. Amoo, T. I. Olawande, T. C. Iruonagbe, A. E. Idowu, and O. A. Adekeye, "Awareness of Breast and Cervical Cancer among Women in the Informal Sector in Nigeria," Open access Macedonian journal of medical sciences, vol. 7, no. 14, p. 2371, 2019. https://doi.org/10.3889/oamjms.2019.259

[14] Z. Omogbadegun, I. Ogundele, and O. Oladipupo, "Applying Neural Network-Based Approach to Sickle Cell Disease-Related Pain Classification," 2019. https://doi.org/10.3844/ jcssp.2019.861.872

[15] F. Kehinde, G. Dedeke, I. Rasaq, and P. O. Isibor, "The Potential of visible light spectra as control measure of Mosquito, the vector of Plasmodium," in IOP Conference Series: Earth and Environmental Science, 2018, vol. 210, no. 1, p. 012009: IOP Publishing. https://doi. org/10.1088/1755-1315/210/1/012009

[16] M. Lee and P. M. Fauchet, "Two-dimensional silicon photonic crystal based biosensing platform for protein detection," Optics Express, vol. 15, pp. 4530-4535, 2007/04/16 2007. https://doi.org/10.1364/oe.15.004530

[17] V. Sharma and V. L. Kalyani, "Nano-cavity coupled waveguide photonic crystal based biosensor detection of cervical cancer using nucleus and cytoplasm," in 2017 International Conference on Information, Communication, Instrumentation and Control (ICICIC), 2017, pp. 1-5. https://doi.org/10.1109/icomicon.2017.8279111

\section{Authors}

Anthony U. Adoghe is a professor of Power Systems with unique interest in applications of engineering to solve medical challenges. E-mail anthony.adoghe@covenant university.edu.ng

Etinosa Noma-Osaghae, is an electronics and telecommunication enthusiast. He has a masters degree in Electronic and Telecommunication and also currently pursuing a $\mathrm{PhD}$ degree in Information and Communication Engineering.

Yabkwa Rimamchika Israel, is a postgraduate student in the Department of Electrical and Information Engineering, Covenant University. E-mail rimamchika.yabkwa @ stu.cu.edu.ng

Article submitted 2019-11-27. Resubmitted 2019-12-12. Final acceptance 2019-12-13. Final version published as submitted by the authors. 